This document is the Accepted Manuscript version of a Published Work that appeared in final form in Analytical Chemistry, copyright (C) American Chemical Society after peer review and technical editing by the publisher. To access the final edited and published work see http://pubs.acs.org/doi/abs/10.1021/acs.analchem.5b01850 


\section{Headspace versus direct immersion solid phase microextraction in complex matrices: investigation of analyte behavior in multicomponent mixtures}

Emanuela Gionfriddo, Érica A. Souza-Silva and Janusz Pawliszyn*

Department of Chemistry, University of Waterloo, Waterloo, Ontario, Canada

"Corresponding author's name: Janusz Pawliszyn

Corresponding author's phone number: 1-519-888-4567 ext. 84641

Corresponding author's fax number: 1-519-746-0435

Corresponding author's e-mail: janusz@sciborg.uwaterloo.ca 


\begin{abstract}
:
This work aims to investigate the behavior of analytes in complex mixtures and matrices with the use of solid-phase microextraction (SPME). Various factors that influence analyte uptake such as coating chemistry, extraction mode, the physicochemical properties of analytes, and matrix complexity were considered. At first, an aqueous system containing analytes bearing different hydrophobicities, molecular weights and chemical functionalities was investigated by using commercially available liquid and solid porous coatings. The differences in the mass transfer mechanisms resulted in a more pronounced occurrence of coating saturation in headspace mode. Contrariwise direct immersion extraction minimizes the occurrence of artefacts related to coating saturation and provides enhanced extraction of polar compounds. In addition, matrix-compatible PDMS-modified solid coatings, characterized by a new morphology that avoids coating fouling, were compared to their non-modified analogous. The obtained results indicate that PDMS-modified coatings reduce artefacts associated with coating saturation, even in headspace mode. This factor, coupled to their matrix compatibility, make the use of direct SPME very practical as a quantification approach and the best choice for metabolomics studies where wide coverage is intended. To further understand the influence on analyte uptake on a system where additional interactions occur due to matrix components, ex vivo and in vivo sampling conditions were simulated using a starch matrix model, with the aim of mimicking plant-derived materials. Our results corroborate the fact that matrix handling can affect analyte/matrix equilibria, with consequent release of high concentrations of previously bound hydrophobic compounds, potentially leading to coating saturation. Direct immersion SPME limited the occurrence of the artefacts, which confirms the suitability of SPME for in vivo applications. These findings shed light into the implementation of in vivo SPME strategies in quantitative metabolomics studies of complex plant-based systems.
\end{abstract}




\section{Introduction}

Since its introduction, the applicability of solid phase microextraction ${ }^{1}$ (SPME) has successfully spread to various fields, including clinical, environmental, and food areas..$^{2-6}$ In recent years, investigations of living systems by use of in vivo SPME strategies have achieved important results for both targeted and untargeted analysis. ${ }^{7-10}$ As a diffusion-based microsampling tool, SPME offers minimum invasiveness of the sampled system, effective strategies for quantitative analysis and, under well-tuned conditions, it is a non-exhaustive extraction technique that causes negligible depletion of the analytes of interest from the matrix. Additionally, in vivo microsampling provides real-time monitoring of the system of interest, capturing unique chemical information related to any changes in matrix composition, alteration of binding equilibria, or biochemical transformations. Solid SPME sorbents have yielded excellent performances due to their suitability for quantitative trace analysis, as well as their ability to efficiently extract compounds within a broad range of molecular masses. ${ }^{11}$ In addition to targeted analysis, solid porous sorbents have been applied for untargeted analysis, such as metabolomics profiling. ${ }^{9,12-14}$ However, extraction from complex matrices can pose significant challenges to the analyst due to the complexity of the matrix itself, the large number of analytes that may be present, and the possible co-extraction of multiple matrix constituents. Generally, solid porous coatings are not suitable for direct immersion in complex media, especially food-based matrices, due to fouling issues. In such cases, the most common practice consists of exposing the SPME coating to the sample headspace (HS) so as to avoid direct contact between the matrix and the coating. ${ }^{15-21}$ The extraction from the HS of complex matrices helps prevent the occurrence of artefacts associated to the attachment of macromolecules to the coating, which can lead to decreased performance resulting from irreversible attachment and thermal decomposition of matrix components when desorption is performed in gas-chromatographic injection port systems. ${ }^{22,23}$ However, despite the advantages of HS sampling for prolonged coating lifetime, this sampling method cannot guarantee proper "balanced coverage" among the analytes extracted; compounds with poor water solubility and good volatility readily enrich the HS of the matrix, whereas more hydrophilic and low volatility compounds, with relatively slower kinetics in solution, suffer higher resistance to transportation to the HS. As such, more volatile compounds are extracted more efficiently than semi-volatiles. Therefore, especially under pre-equilibrium conditions, the HS extraction of complex matrices involving semi-volatile and polar compounds is not always very efficient. The extraction efficiency for polar and semi-volatile compounds improves drastically when direct exposure of the coating to the matrix is performed, as the diffusion coefficients through the matrix, which define the mass transfer properties of the extraction mode, are 
similar for all small molecules present in the system. As a result, a more comprehensive analyte coverage $^{24}$ is obtained. Therefore, the direct immersion (DI) strategy is very promising for broad untargeted screening of analytes present in the matrix, such as for metabolomics profiling by in vivo sampling. Moreover, the capability of SPME to extract compounds in their free concentration makes the technique sensitive to changes in matrix composition and alterations in binding equilibria. Hence, SPME constitutes a useful tool for determination of binding constants of target analytes to macromolecules (e.g. proteins), investigations of complex system composition in response to internal/external stimuli, and monitoring of chemical transformations (e.g. pharmacokinetics studies) ${ }^{25-30}$. As discussed above, despite the undisputable advantages presented in terms of extraction efficiency, solid porous coatings show certain drawbacks associated with deterioration of the coating surface due to direct exposure to complex matrices for extended periods of time. ${ }^{22}$ To overcome this limitation of solid sorbents, matrix-compatible SPME coatings were developed. In this approach, a smooth thin layer of PDMS surrounding the solid adsorbent prevents the adhesion of matrix components, thus avoiding fouling of the coating. These modified coatings were noted to be suitable for extended series of extractions irrespective of the direct exposure of the extraction phase in the food matrix..$^{22}$ Another characteristic of the solid porous coating is the possible surface saturation that may occur due to adsorption from matrices containing a high concentration of target analytes and/or interferences. When saturation takes place, non-linear adsorption isotherms occur as a result of competitive extraction phenomenon; ${ }^{31}$ compounds bearing stronger affinity for the coating begin to gradually displace compounds with less affinity for the extraction phase. The competitive adsorption phenomenon has been reported for HS ex vivo analysis of different food matrices $^{15,16}$, while no artefacts related to coating saturation were observed for analysis of complex matrices in direct immersion (DI) batch extractions and in vivo applications. ${ }^{2}$ This could be attributed to the fact that under physiological conditions, the matrix components (such as suspended solids and macromolecules) significantly bind the analytes, especially non-polar compounds, reducing their free concentration and their availability to the coating. In this context, this work aims to contribute to a better understanding of the behavior of analytes in complex multi-component systems, as well as the mechanics of saturation and competitive adsorption phenomena, by comparing different sampling approaches, including simulated in vivo conditions. The present study is of particular importance to food investigations, especially untargeted analysis, as it involves the optimization of coating and extraction conditions for best analyte coverage and accuracy of results. Moreover, a simulation of different extraction modes applied to a complex matrix shows that the occurrence of coating saturation is strictly dependent on matrix composition and binding equilibria, which can be easily affected by sample handling and pre-treatment. In addition, the results affirm the observation that the SPME sampling technique reflects the metabolome under study. 


\section{EXPERIMENTAL SECTION}

Chemicals and Materials The details for chemicals and materials are described in Supporting Information (section 1.1). Commercial SPME fiber assemblies in 23-gauge needle sizes and automated formats, $100 \mu \mathrm{m}$ PDMS (stable flex), $65 \mu \mathrm{m}$ PDMS/DVB (stableflex), $85 \mu \mathrm{m}$ Car/PDMS (stableflex), and 50/30 $\mu \mathrm{m}$ DVB/Car/PDMS (stableflex), were purchased from Supelco (Oakville, Canada). PDMSmodified fibers were obtained by modifying commercial coatings (PDMS/DVB and DVB/Car/PDMS) according to the procedure described by Souza-Silva et al. ${ }^{22}$

Standards and Samples Preparation Details related to the preparation of standard solutions are described in Supporting Information (section 1.2). With the aim of covering a wide range of functionalities and chemical properties, the following model compounds were chosen: 1-pentanol, 2hexanoate, ethyl butanoate, benzaldehyde, acetophenone, benzene, eucalyptol, a-pinene, 2-undecanone, ethyl nonanoate, 1-undecanol. The concentration of each analyte in the mixture was set to guarantee enough sensitivity for all analytes with all tested coatings, both in DI and HS extraction modes. A summary of the probe compounds, with their physicochemical characteristics and the quantitation ions, is shown in Supporting Information Table S1. In addition, a detailed description of the probe analytes selected for this investigation is given in Supplementary Information (section 1.3).

Preparation of Starch Dispersions and Gels Details related to the preparation of starch dispersions and gels are described in Supporting Information (section 1.4).

SPME Procedure Samples were freshly prepared and analyzed immediately in order to avoid any possible degradation of the compounds. Extraction temperature and agitation speed were set at $30{ }^{\circ} \mathrm{C}$ and $500 \mathrm{rpm}$, respectively. The extraction times evaluated were 15, 30 and $60 \mathrm{~min}$; the incubation time was set at 0 , 2, and $20 \mathrm{~min}$ for DI-static (no agitation), DI-dynamic (with agitation), and HS extractions, respectively. For HS extractions, the volume of HS was kept constant throughout so as to avoid the concentration or dilution of analytes in the HS. For all tested coatings, desorption in the injector port of the GC-MS was maintained at $10 \mathrm{~min}$ in splitless mode and at the maximum coating temperature recommended by the manufacturer. Desorption efficiency was tested by re-injection of the coatings immediately after the analysis, keeping the coating sealed at $4^{\circ} \mathrm{C}$ prior to the injection.

Instrumentation Details on analytical instrumentation are shown in Supporting information (section 1.5)

\section{RESULT AND DISCUSSION}

Behavior of solid porous and liquid SPME coatings in multi-analytes systems: effect of coating chemistry, analyte affinity, and extraction mode 
Due to the aqueous nature of plant materials, as is the case for most biological matrices, an initial assessment of analyte behavior in a multi-component mixture was performed in aqueous solution. This model system provided an adequate platform to investigate the behavior of the coatings and evaluate competitive adsorption phenomena in a multi-component system. Moreover, saturation of the coating was purposely induced by introducing a high concentration of a compound having high affinity towards the coating. Accordingly, extractions were independently performed in HS and DI modes from an aqueous mixture containing the probe analytes (Table S1). The compound selected to induce coating saturation was $\alpha$-pinene (Henry's constant, $1.07 * 10^{-1} \mathrm{~atm} * \mathrm{~m}^{3} / \mathrm{mol},{ }^{32}$ and with high affinity for the coating). For the first set of extractions, $\alpha$-pinene was kept at a low concentration $\left(8.3 \mu \mathrm{g} \mathrm{L}^{-1}\right)$ in order to avoid any kind of saturation and/or displacement phenomena. In addition, two other sets of extractions were carried out at two higher levels of $\alpha$-pinene concentrations, approximately 10- and 100-fold of the initial $\alpha$-pinene concentration in the mixture. In a fourth set of extractions, the concentration of $\alpha$-pinene was maintained above its solubility (4.07 $\mathrm{mg} \mathrm{L}^{-1}$ ) in water. The concentration of the saturating compound was set as to purposely induce saturation of the coating, providing a clear visualization of the competitive adsorption phenomena. Although such high concentrations of $\alpha$-pinene are not expected under physiological conditions during in vivo measurements, they might be created when the tissue of the plant or fruit is crushed, releasing compounds from storage compartments such as organelles or micelle-like substructures. $^{33}$ Since all conditions were kept constant, except for the concentration of $\alpha$-pinene, it was assumed that inter-analyte displacement did not take place if the amount extracted for each analyte was statistically the same (within \pm SD) compared to the amount extracted at the lowest level of spiked $\alpha$ pinene $\left(8.3 \mu \mathrm{g} \mathrm{L}^{-1}\right)$. Although inter-analyte competitive adsorption has been modeled for a two-component system by our group ${ }^{34}$, general considerations can also be drawn for complex matrices and multicomponent systems (Supplementary Information Section 1.6). Figure 1 shows results corresponding to the amounts of probe analytes extracted at varying concentrations of $\alpha$-pinene using three solid porous coatings (PDMS/DVB, Car/PDMS, DVB/Car/PDMS) and a liquid coating (PDMS $100 \mu \mathrm{m}$ ) in DI mode. 
PDMS $100 \mu \mathrm{m}$

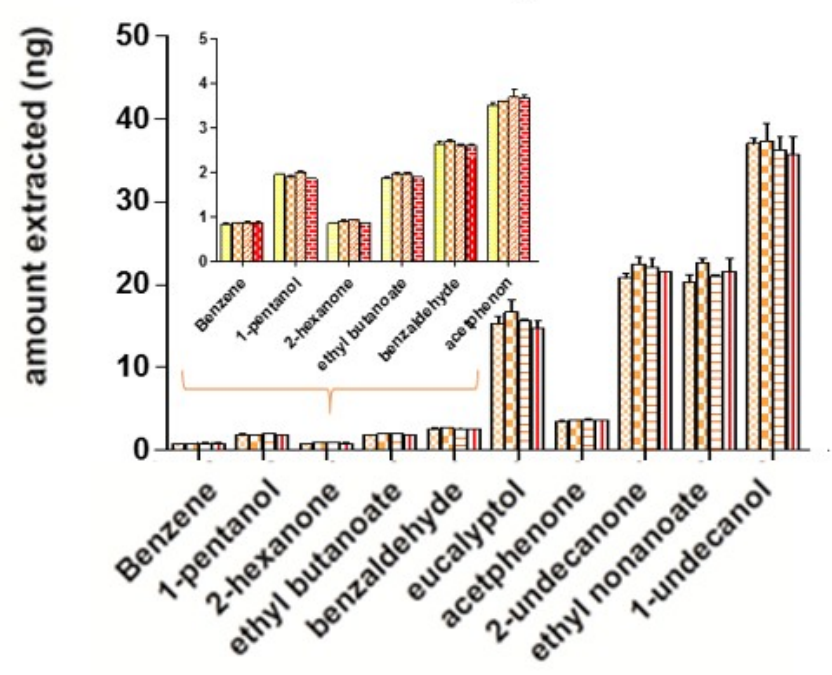

DVB/Car/PDMS

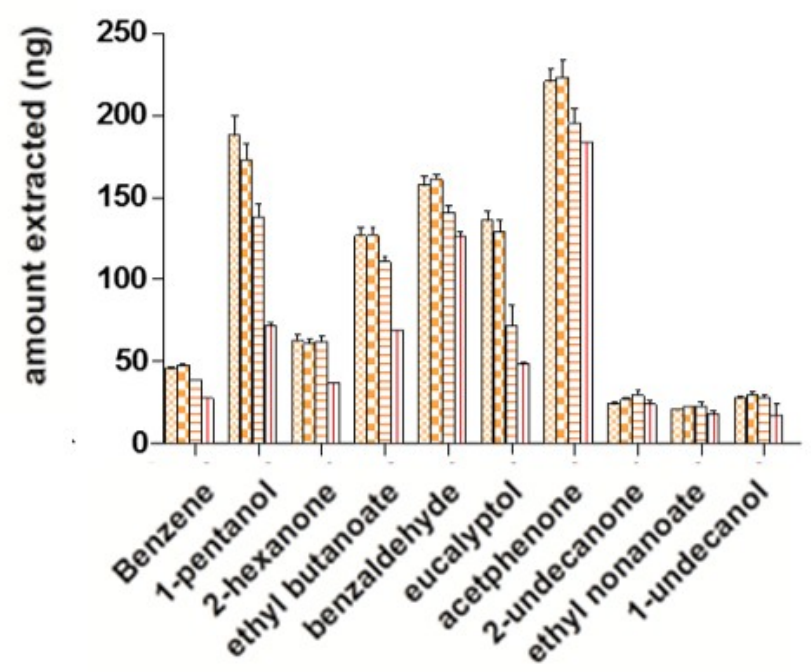

PDMS/DVB

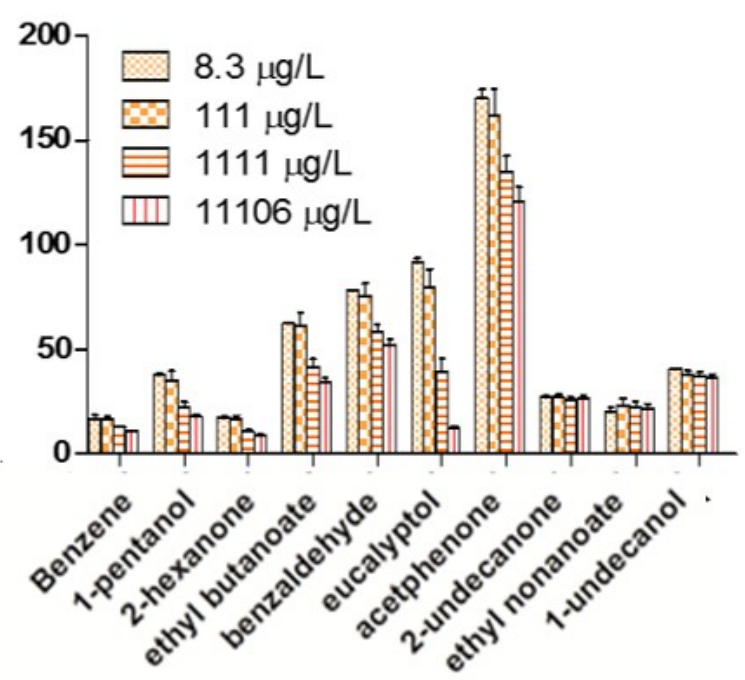

Car/PDMS

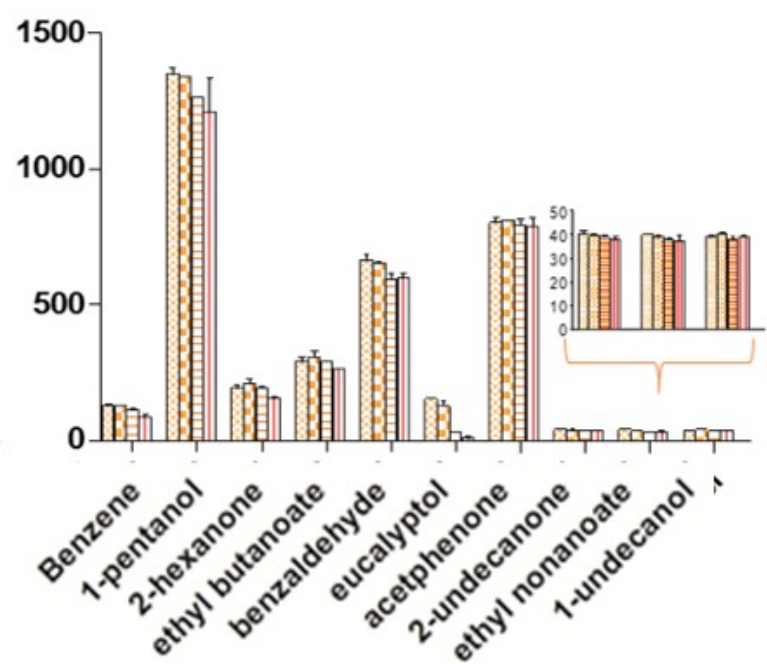

Figure 1: Comparison of extracted amounts for three solid porous SPME coating (PDMS/DVB, DVB/Car/PDMS, $\mathrm{Car} / \mathrm{PDMS}$ ) and one liquid (PDMS) commercial SPME coating, after 60 minutes (at $35^{\circ} \mathrm{C}$ and 500rpm) of direct exposure to an aqueous solution containing varying concentrations of $\alpha$-pinene and constant concentrations of analytes (benzene $14.9 \mu \mathrm{g} \mathrm{L}^{-1}$, 1-pentanol $664 \mu \mathrm{g} \mathrm{L} \mathrm{L}^{-1}$, 2-hexanone and ethyl butanoate $66.5 \mu \mathrm{g} \mathrm{L}^{-1}$, eucalyptol 83.1 $\mu g \mathrm{~L}^{-1}$, acetophenone and benzaldehyde $133 \mu \mathrm{g} \mathrm{L}^{-1}$, 2-undecanone, ethyl nonanoate, and 1-undecanol $6.6 \mu \mathrm{g} \mathrm{L}^{-1}$ ). Error bars represent \pm standard deviations obtained for three replicates.

It is worth nothing that for the PDMS $100 \mu \mathrm{m}$ coating, the extracted amount of analytes did not show dependence on $\alpha$-pinene concentration at each spiked concentration level. This observation supports the concept that liquid coatings are not characterized by competitive adsorption, and as such, for this system it suggests: $i$ ) the coating is not affected by swelling at the investigated analyte concentration, and ii) the 
analytes in the mixture are stable throughout the extraction process, even at high $\alpha$-pinene concentrations, suggesting that no analyte degradation or side reaction affects the system investigated. The latter information was critical to ensure that any changes observed in the probe analyte amounts extracted by the solid porous coatings were due exclusively to the changes in the concentration of $\alpha$-pinene, indicating that they were solely related to competitive adsorption of the analytes. Results for the PDMS coating in Figure 1 also demonstrated that it displayed less sensitivity compared to other solid porous coatings, particularly for polar analytes. As such, the use of liquid coatings for screening of complex matrices that include a broad range of analytes with different polarities is not recommended. The results (Figure 1) demonstrate also that the Car/PDMS is less prone to artefacts related to coating saturation compared to PDMS/DVB and DVB/Car/PDMS under the same working conditions. This can be explained by considering the specific surface area of the polymer and the coating characteristics. Carboxen 1006 particles bear a higher specific surface area compared to DVB and, in addition, Car/PDMS has a greater total phase volume than PDMS/DVB. ${ }^{11}$ In view of this, more active sites are available for adsorption for the Car/PDMS than for the PDMS/DVB coating. Therefore, the amount of $\alpha$-pinene extracted during the experiment is far from reaching the capacity of the coating. Furthermore, Carboxen 1006 is constituted by narrow micropores small enough to accommodate smaller analytes, and thus, tends to have a higher affinity for low molecular weight and polar compounds compared to PDMS/DVB. This implies that saturation and consequent inter-analyte competitive adsorption from the Carboxen coating is less likely to occur. Therefore, in addition to the concentration of the displacing compound ( $\alpha$-pinene), the affinity of all the compounds in the system for the extraction phase plays a significant role in the occurrence of the phenomena. Although the affinity of the compounds may be insignificant for shorter extraction times (pre-equilibrium conditions), this becomes prominent for longer extraction times. Thus, in this study, despite its relatively higher affinity for the Car/PDMS, $\alpha$-pinene did not induce displacement of during the 60 minute extraction because it was still under pre-equilibrium conditions. However, at longer extraction times beyond $60 \mathrm{~min}$, significant displacement for all the analytes may be noticed even for Car/PDMS because of its solid porous characteristic. Generally, as shown in Figure 1, as the concentration of $\alpha$-pinene in the mixture increases, displacement of the analytes takes place. In fact, for an $\alpha$-pinene concentration above $1 \mathrm{mg} \mathrm{L}^{-1}$, the amount extracted for some analytes was significantly diminished when compared to the amount extracted from the mixture containing $\alpha$-pinene at its lowest level $\left(8.3 \mu \mathrm{g} \mathrm{L}^{-1}\right)$. Trends in analyte displacement were similar for PDMS/DVB and DVB/Car/PDMS, although the extracted amounts were different. The DVB/Car/PDMS coating is constituted by an inner layer of Carboxen that is overcoated by a thicker outer layer of DVB particles. This design allows larger molecules first to interact with the outer and weaker adsorbent DVB, while the small molecules diffuse through but get adsorbed by Carboxen 1006 particles that represent the stronger adsorbent. ${ }^{35}$ The 
PDMS/DVB coating contains DVB particles coated using liquid PDMS as glue. The similar displacement pattern observed for these two coatings also for HS sampling (Table S2) may be attributed to the overall phase volume of the DVB layer and their total surface area. In both coatings, the DVB layers have nearly the same surface area, which is about 8x larger than that of the Carboxen layer in DVB/Car/PDMS. From the data obtained, the DVB layer will perform predominantly as the adsorptive layer, which explains the similarity in the displacement trend observed for these coatings.

In addition, the obtained results clearly show (Figure 1) that eucalyptol (a terpene), was most readily displaced by $\alpha$-pinene (another terpene) for all three coatings, even though it does not bear the lowest affinity for the extraction phases among the investigated analytes. On the other hand, for coatings containing DVB sorbent, 1-pentanol, ethyl butanoate, benzaldehyde, and acetophenon were readily affected by an increasing concentration of $\alpha$-pinene, indicating that these analyte structures interact with the active surface of the DVB coating in a location similar to that of $\alpha$-pinene. It would appear that there is no random heterogeneity of sorbent surface, but rather, well-defined sub-structures that create preferred sorption sites for molecules having structures similar to eucalyptol. As such, it can be concluded that alike molecules with stronger affinity by the sorbent will preferentially displace those with alike structure and with less affinity. In light of this, it can be proposed that the characteristics of the DVB surface are different compared to Carboxen, as this effect is not observed for that later sorbent. Although this line of inquiry is out of the scope of the present work, the observed phenomenon is under further investigation to confirm the potential of SPME as an effective tool to characterize the molecular recognition properties of solid surfaces. This will be important not only to study the characteristics of extraction sorbents, as discussed above, but also to shed further insight into the behavior of natural solids present in complex matrices that influence the free concentration of target analytes based on their adsorption properties ${ }^{33,36}$. Extraction mode is also a critical parameter that influences the occurrence of coating saturation on solid porous coatings. Generally, the main difference between extraction in HS and DI mode is the mass transfer rate. In the case of HS extraction, the mass transfer rate is proportional to the Henry`s constants of the analytes under study, whereas in the case of DI extraction, it is correlated to the unbound analyte fraction (free concentration). Figure S1 shows a comparison in competitive adsorption occurrence at the same working conditions for HS and DI extraction performed in an aqueous solution by PDMS/DVB sorbent. The results clearly show an increased occurrence of competitive adsorption in HS mode at lower amounts of spiked $\alpha$-pinene compared to DI, and even shorter extraction times (15 minutes). Generally, comparing DI and HS modes, competitive adsorption was significant for all HS mode extractions starting from $111 \mu \mathrm{g} \mathrm{L}^{-1}$ of spiked $\alpha$-pinene. For example, in HS extraction mode, the displaced amount ranged from 19 to $23 \%$ for the analytes in the mixture, whereas at the same concentration of $\alpha$-pinene (111 $\mu g \mathrm{~L}^{-}$ 
${ }^{1}$ ), competitive adsorption was negligible in DI mode. An explanation can be proposed taking into consideration the mass transfer of analytes occurring within the system. The high Henry's Law constant of $\alpha$-pinene and possible matrix modification (e.g. salting out effects) make it readily available in the headspace of the sample, where the diffusion coefficients are orders of magnitude higher than those in the solution. For this reason, the faster adsorption rate in the HS, together with the high affinity of $\alpha$-pinene for the coating, yields high amounts of $\alpha$-pinene being extracted even at shorter extraction times in HS mode. On the other hand, for DI extractions, the kinetics of diffusion for $\alpha$-pinene in the bulk of the solution is slower in the matrix. Moreover, the mass transfer resistance of the boundary layer surrounding the coating in the aqueous phase compared to gas is much larger, since the diffusion coefficient in water is four orders of magnitude smaller compared to air. This implies that at shorter extraction times, the amount of $\alpha$-pinene extracted by HS is higher than that extracted by DI ( $\approx 39 \mu \mathrm{g} v s \approx 24 \mu \mathrm{g}$ at $15 \mathrm{~min}$ in HS and DI, respectively); thus, when saturation of the coating occurs, displacement phenomena are more evident in HS mode.

Analytes that deserve particular note are 2-undecanoate, ethyl nonanoate, and 1-undecanol: due to their similar hydrophobicity and/or affinity for the coating, compared to $\alpha$-pinene, they are not affected by coating saturation regardless of the extraction mode used. This implies that their extracted amount is constant regardless of the increasing amount of $\alpha$-pinene spiked into the mixture. In summary, coating saturation occurs more pronouncedly in HS than in DI mode; as such, displacing compounds with higher coating affinity can cause displacement to occur to a significant extent, even at relatively short extraction times in HS mode, although this may be avoided in DI mode.

DVB/Car/PDMS and Car/PDMS also showed similar trends in terms of extraction mode, however, given the previous discussion regarding the intrinsic peculiarities of carboxen and DVB sorbents, the extent of displacement and the differences noticed between the two extractions modes are far less pronounced. Table S3 summarizes representative results for DVB/Car/PDMS and Car/PDMS.

\section{Comparison of PDMS-modified coatings and their commercial analogues towards saturation and competitive adsorption occurrence}

Recently, in order to overcome issues related to the compatibility of solid porous coatings to DI-SPMEGC analysis of complex food matrices, matrix-compatible coatings were developed. ${ }^{22}$ Because of the proven suitability of the PDMS-modified solid coatings for DI extraction in complex food matrices, these coatings are appropriate for direct monitoring in living systems, especially for metabolomics investigation purposes. For this reason, the new matrix-compatible coatings were also investigated for competitive adsorption occurrence. Two different PDMS-modified coatings (PDMS-PDMS/DVB and PDMSDVB/Car/PDMS), were tested at 15, 30, and $60 \mathrm{~min}$ in HS and DI modes, at the same previously 
mentioned working conditions in aqueous samples. As can be deduced from Figure 2, the competitive adsorption occurrence was far less pronounced for the PDMS-modified coatings. This observation may be attributed to two synergistic phenomena:

1. The slower kinetics of extraction for the PDMS-modified coating due to the presence of the thin PDMS layer surrounding the solid porous polymer, which constitutes a diffusion barrier for analytes before reaching the adsorbent (Figures 2 and S2).

2. PDMS-modified coatings provide additional coating capacity to concentrate more hydrophobic compounds, therefore decreasing the amount of analyte reaching the solid porous sorbent.

DI -60 min- PDMS/DVB

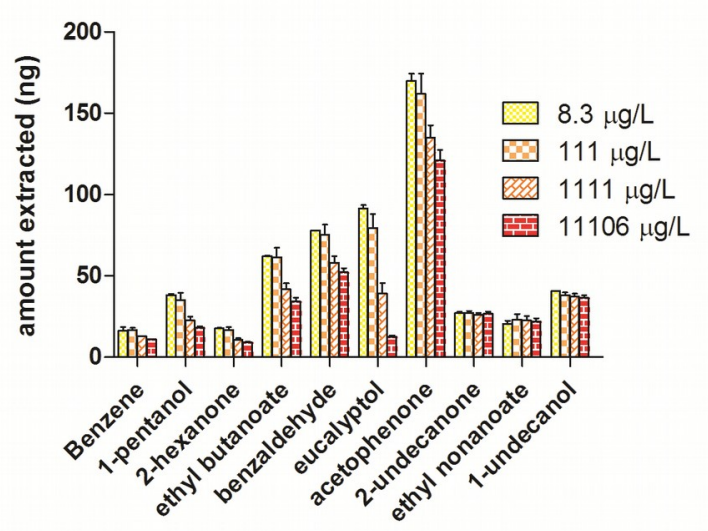

DI- 60 min-DVB/Car/PDMS

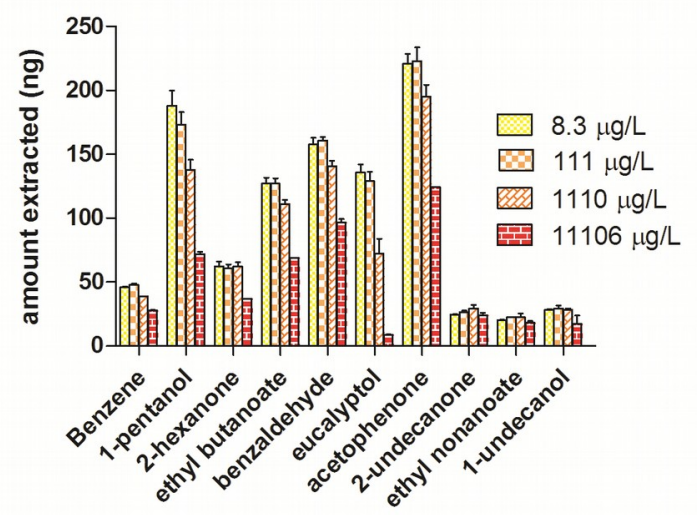

DI- 60 min- PDMS-modified PDMS/DVB

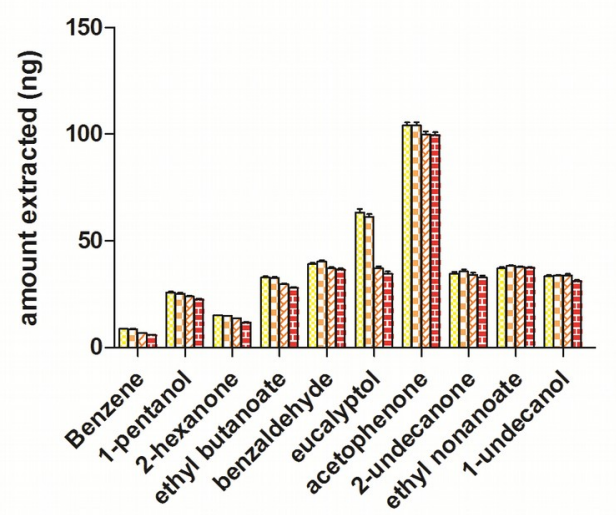

DI- 60 min-PDMS modified DVB/Car/PDMS

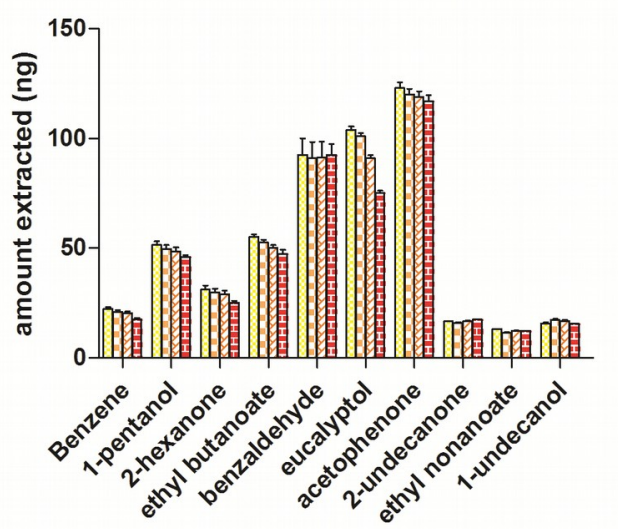

Figure 2: Comparison of commercial coatings and PDMS-modified coatings for displacement occurrence in direct immersion analysis of water samples. Error bars represent standard deviations obtained for three replicates. Analyte concentrations in aqueous solution were set as: benzene $14.9 \mu \mathrm{g} \mathrm{L}^{-1}$, 1-pentanol $664 \mu \mathrm{g} \mathrm{L}{ }^{-1}$, 2-hexanone and ethyl butanoate $66.5 \mu \mathrm{g} \mathrm{L}^{-1}$, eucalyptol $83.1 \mu \mathrm{g} \mathrm{L} \mathrm{L}^{-1}$, acetophenone and benzaldehyde $133 \mu \mathrm{g} \mathrm{L}^{-1}$, 2-undecanone, ethyl nonanoate and 1-undecanol $6.6 \mu \mathrm{g} \mathrm{L} \mathrm{L}^{-1}$. Extraction were performed for 60 minutes at $500 \mathrm{rpm}$ and at $35^{\circ} \mathrm{C}$. 
In fact, the later hypothesis is confirmed by the observation that in addition to a substantial observed decrease in the displacement effect, the amount of $\alpha$-pinene extracted with the PDMS-modified coating was more than $50 \%$ higher than that extracted by its non-modified equivalent. This indicates that the PDMS layer preconcentrates $\alpha$-pinene while preventing the saturation of the DVB layer. The results of this study show that the enhanced capacity of the PDMS-modified coating may result in the reduction of inter-analyte displacement phenomena as compared to commercial solid coatings at the same working conditions.

Interestingly, as shown in Supporting Information Figure S2, the PDMS-modified coatings consistently showed significantly less displacement, even in HS and longer extraction times, when compared with the corresponding non-modified analogous.

\section{Evaluation of matrix effects using a starch based food model}

SPME, as a non-exhaustive technique, only extracts the free form of analytes. In cases of complex matrices where binding is more pronounced, the free concentration of analytes is significantly lowered compared to spikes of the same concentration in water. This occurs because of the binding affinity of the analytes for the macromolecules and/or dispersed solids in the matrix. That is, more hydrophobic analytes tend to bind strongly to matrix components, lowering their free-concentration in the matrix and their availability for SPME extraction. On the other hand, binding equilibria offers the effect of "balanced coverage” since polar analytes having lower affinity for the coating become relatively abundant in their free form, compared to non-polar components, which usually have more affinity for the extraction phase, but are found in lower free concentrations. Analyte/matrix interactions are mainly characterized by nonspecific and reversible binding forces and can be altered by sample treatment (e.g. disruption of the matrix, changes in ionic strength and temperature), alteration of binding equilibria (e.g. related to protein precipitation, unfolding or disruption of biological compartments), and activation of enzymatic activities $26,28,37$. Such matrix modifications often improve the extractability of the analytes by enhancing their availability to SPME coatings, which sometimes may lead to saturation and displacement effects. However, when sampling is performed in well-regulated, non-disturbed systems where there is no matrix modification (such as in vivo sampling), artefacts related to coating saturation may also be absent, as binding of components within the matrix limits the free concentration of the most hydrophobic analytes, resulting in negligible matrix or coating saturation. Indeed, under such conditions, SPME becomes a proper tool to conduct experiments devoted to the understanding of natural systems in their physiological state, including responses to external/internal stimuli that may cause alterations in the ratio of free/bound concentrations of investigated analytes. This can be attributed to the miniaturized design of the extraction phase, which literally maintains the "physiological integrity" of the system investigated. ${ }^{30,38}$ The 
aforementioned characteristics, among others, are responsible for the successful implementation of SPME in metabolomics studies applied to plant-based systems, but also to other living organisms ${ }^{10,39,40}$. After assessing the solid porous coatings and their behavior towards saturation and competitive adsorption in aqueous medium (with no solids matrix components), further experiments were carried out using a starch gel system as a model-matrix that closely mimics the texture consistency of fruits and vegetables. This approach simulates well, and in a broader context, the binding characteristics of the matrix and the freeconcentration of analytes. Indeed, starch gel systems are largely used as a food models in studies related to aroma release. ${ }^{41-44}$ The model-matrix chosen in our study is suitable to simulate the encapsulation and binding of hydrophobic compounds in native biological compartments present in vegetable tissues due to the presence of proteins, native lipids, and amylopectins. ${ }^{45,46}$ SPME has been already successfully used for binding constant determinations in complex matrices, and is a technique that often reflects the molecular state of a biological system. ${ }^{26,28,30}$ In line with our study objective, saturation of the system was again induced by spiking high concentrations of $\alpha$-pinene. The evaluation of analyte behavior in a complex matrix is of critical importance, as the presence of macromolecules and different binding phases affect the amount of unbounded analytes (free concentration). For all the experiments carried out with starch matrix, a readjustment of the spiked amount for some of the analytes was necessary (compared to previous experiments in pure water) in order to guarantee enough sensitivity (benzene $33.2 \mu \mathrm{g} \mathrm{kg}^{-1-}$, 2undecanone, ethyl nonanoate and 1-undecanol $166.1 \mu \mathrm{g} \mathrm{kg}^{-1}$, $\alpha$-pinene 166.11, 332.2, 1662.7 and 16627.9 $\mu \mathrm{gg}^{-1}$ ). With the chosen model and set up, three extraction modes and sample preparation techniques frequently used in SPME for food analysis were simulated:

a. Headspace (HS) extractions: performed by crushing the matrix and extracting from the HS while agitating the sample.

b. Dynamic Direct Immersion (D-DI): this extraction mode simulates ex vivo batch analysis of food where the sample is crushed and transferred into an appropriate vial, and where the coating is directly exposed in the matrix bulk under agitated conditions.

c. Static Direct Immersion (S-DI): this extraction mode simulates in vivo extractions in biological systems where the tissue is not disrupted and the extraction is performed in static conditions without any matrix modification.

Testing of these three extraction modes was critical to understanding analyte behavior in complex matrices due to the various mass transfer mechanisms that drive the extraction process and the alteration of the analytes/matrix binding equilibria. For HS extractions, it is important to note that generally, the major rate-limiting step is the diffusion of the analytes from the bulk of the matrix to the HS. As per 
common practice in sample preparation for HS analysis, our starch-gel food model system was disrupted before exposure of the coating. This process induces the release of the entrapped compounds, mostly hydrophobic, to readily enrich the HS. Thus, under pre-equilibrium extraction conditions, analytes will be selectively transferred into the HS. In other words, the HS is largely enriched with more hydrophobic and volatile compounds, while the aqueous content still holds a significant amount of polar compounds. When high Henry's law constants for given compounds are not only associated with rapid enrichment of the HS, but also with high partition into the extraction phase, the synergy of these two effects straightforwardly induces coating saturation, and thus the occurrence of competitive adsorption.

Conversely, for the D-DI setup, the coating is directly exposed to the bulk of the matrix, and the overall mass transfer to the extracting phase is limited by the diffusion of the analytes through the boundary layer surrounding the coating. The thickness of the boundary layer largely depends on the degree of agitation and the nature of the matrix. The relevance of the gel-system was to provide the necessary tortuosity that simulates an actual plant-based matrix, as this plays an important role in the mobility of the analytes. Thus, with agitation, overall migration of the analytes through the bulk of the matrix will be significantly improved. D-DI mode also involves the disruption of tissue before the extraction process. Thus, enhanced mixing of the analytes occurs due to the rupture of the amylose helices and other possible aggregates formed with amylopectines, proteins, and native lipids, as well as alteration of binding equilibria. Contrary to the fiber-headspace-matrix system, the diffusion of small molecular weight hydrophilic compounds will be significantly improved. The relevance of this phenomena can be seen in the obtained results; as shown in Figure 3, a more balanced coverage of the analytes even in pre-equilibrium conditions was obtained. 

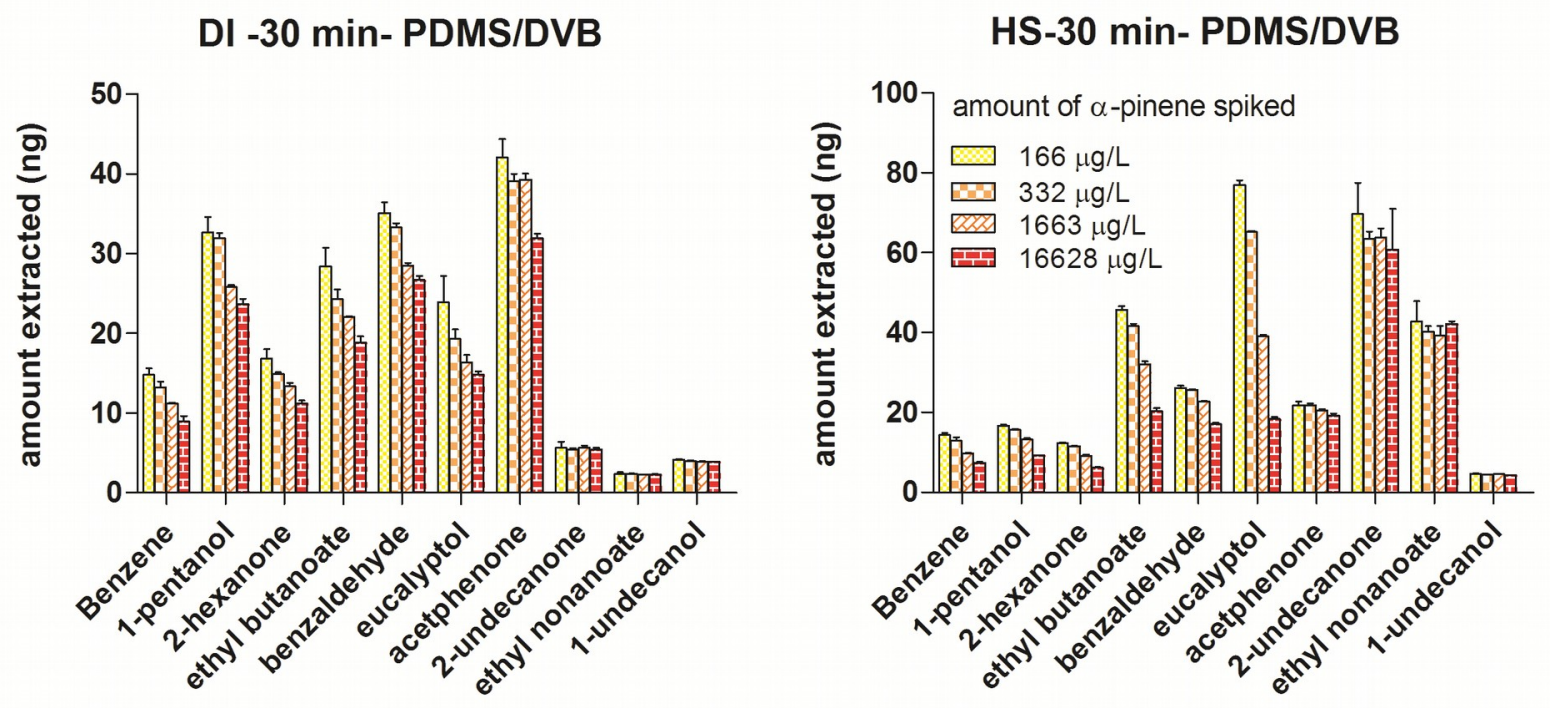

Figure 3: Profiles of analyte uptake in direct immersion and headspace modes under pre-equilibrium extraction time (30 minutes) in a starch gel system under agitation conditions (500rpm). Extractions were carried out at $35^{\circ} \mathrm{C}$ for 30 minutes in direct immersion and headspace mode from starch gel enriched with the following amounts of analytes: benzene $33.2 \mu \mathrm{g} \mathrm{kg}^{-1}$-,1-pentanol $665 \mu \mathrm{g} \mathrm{kg}^{-1}$, 2-hexanone and ethyl butanoate $66.5 \mu \mathrm{g} \mathrm{kg}^{-1}$, eucalyptol $83.1 \mu \mathrm{g} \mathrm{kg}^{-1}$, acetophenon and benzaldehyde $133 \mu \mathrm{gg}^{-1}$ 2-undecanone, ethyl nonanoate and 1-undecanol $166 \mu \mathrm{g} \mathrm{kg}^{-1}, \alpha$-pinene $166,332,1663$ and $16628 \mu \mathrm{g} \mathrm{kg}^{-1}$

As seen in Figure 3, generally, there is an improvement in the extraction of polar analytes for DI vs HS extractions, which is in agreement with SPME fundamentals, as well as recent results obtained in a comparative metabolomics study of apples. ${ }^{24}$ This outcome is associated with the difference of free concentrations between polar (mostly existing as free fractions in solution) and hydrophobic analytes (mostly bound to matrix). This implies that DI extraction from living systems provides the best strategy to achieve balanced coverage of the analytes extracted in global untargeted metabolomics studies. The S-DI mode was carried out to simulate a typical in vivo extraction where external agitation influence is negligible. The static DI mode is characterized by the slowest mass transfer kinetics among the three sampling modes tested. When in vivo analysis is performed on plant-based systems, the entire biological environment surrounding the coating can be considered to be static. Often in this mode, the structural integrity of the plant-based living system may not be perturbed. Thus, in order to mimic in vivo extraction conditions, any perturbation of the starch gel system after submitting it to a cooling procedure was avoided, and the extraction carried out in static conditions. 
To assess the system under equilibrium and pre-equilibrium conditions for all the tested extraction modes, extraction time profiles were acquired using a $2.5 \%$ of starch $(w / w)$ at $\mathrm{pH}$ of 6.8 , up to 24 hours. Equilibrium was achieved at 6 hours; pre-equilibrium extraction time was set at 30 minutes. Further sets of extractions were performed in HS, D-DI, and S-DI modes, according to the same saturation-inducing procedure described in previous sections.

S-DI- 30 min- PDMS/DVB

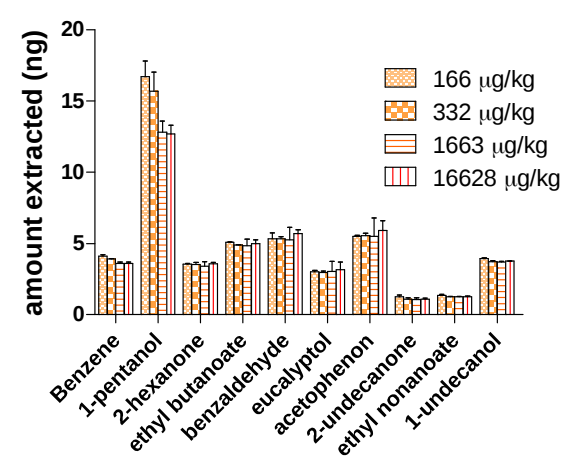

S-DI-6h- PDMSIDVB

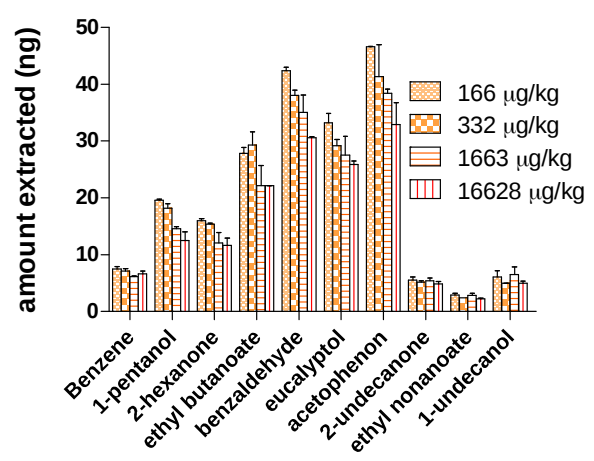

D-DI -30 min- PDMS/DVB
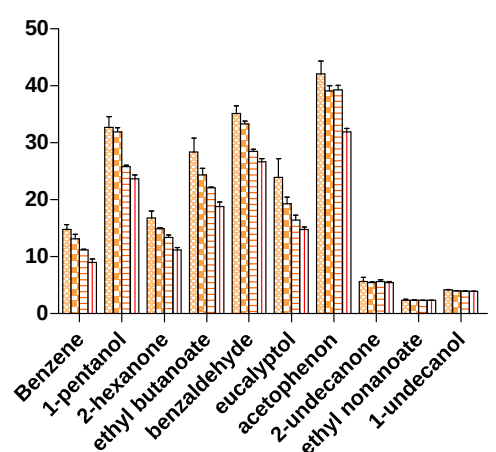

D-DI-6h- PDMS/DVB

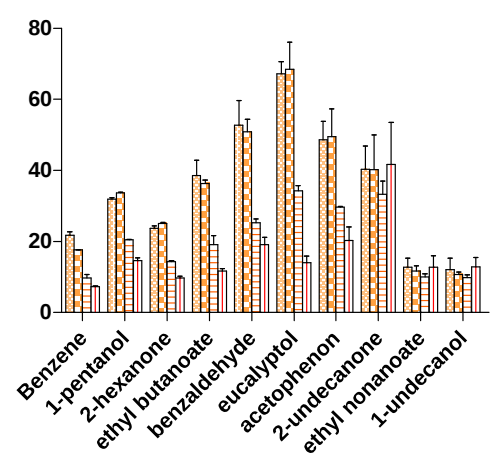

HS-30 min- PDMS/DVB

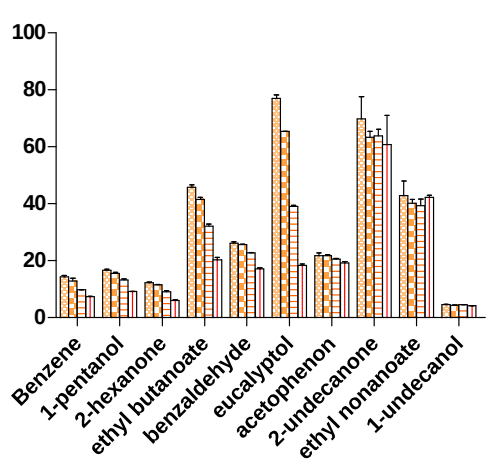

HS-6h- PDMS/DVB

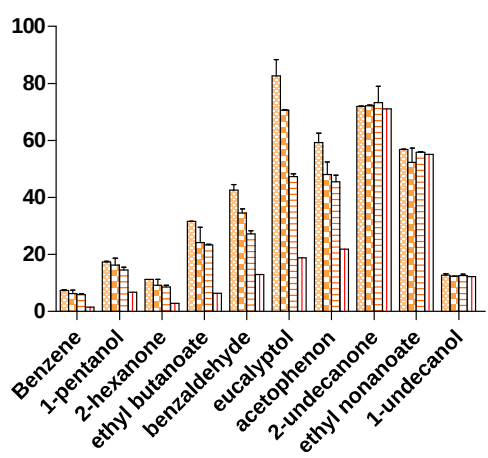

Figure 4: Comparison of amounts extracted at different extraction modes at pre-equilibrium (30 minutes) and equilibrium (6 hours) conditions for starch gel system $2.5 \%(\mathrm{w} / \mathrm{w})$. Starch gel was spiked at the following concentrations: benzene $33.2 \mu \mathrm{g} \mathrm{kg}^{-1}$,1-pentanol $665 \mu \mathrm{g} \mathrm{kg}{ }^{-1}$, 2-hexanone and ethyl butanoate $66.5 \mu \mathrm{gg}^{-1}$, eucalyptol $83.1 \mu \mathrm{g} \mathrm{kg}^{-1}$, acetophenon and benzaldehyde $133 \mu \mathrm{g} \mathrm{kg} \mathrm{\textrm {L } ^ { - 1 }}$ 2-undecanone, ethyl nonanoate and 1undecanol $166 \mu \mathrm{g} \mathrm{kg}^{-1}$, $\alpha$-pinene 166, 332, 1663 and $16628 \mu \mathrm{g} \mathrm{kg}{ }^{-1}$. Extraction were performed at $35^{\circ} \mathrm{C}$, for extraction in D-DI and HS agitation of 500 rpm was used.

In agreement with previous findings reported in this work, Figure 4 shows that displacement was more prominent in HS mode, even at pre-equilibrium conditions, due to the facilitated HS enrichment and faster uptake of hydrophobic compounds on the coating. The disruption of the gel-system causes a larger 
release of hydrophobic compounds into the HS of the matrix, thus enhancing the displacement phenomena. The results herein presented show that in order to prevent or minimize the occurrence of the displacement phenomena and ensure unbiased data acquisition, S-DI extraction mode is the most appropriate mode for metabolomics studies of complex matrices. S-DI offers the least inter-analyte competitive adsorption as compared to the other extraction modes. In the case of HS and D-DI extraction modes, although there is improvement in the mass transfer kinetics of the system, this leads to an increase in the overall amount of analytes extracted. As such, the active sites of the extraction phase may become significantly occupied, leading to potential displacement as extraction continues for a longer period of time. However, under static mode, the slow mass transfer limits the amount of analytes extracted within pre-equilibrium extraction times. The S-DI mode simulates a typical in vivo system where the physiological state of the system is not perturbed, showing a drastic reduction on inter-analyte displacement phenomenon as compared to other extraction modes in both pre-equilibrium and equilibrium conditions. From the data presented in figure S3, even at equilibrium conditions and with $\alpha$ pinene spiked at the highest tested concentration $\left(16628 \mu \mathrm{g} \mathrm{kg}^{-1}\right)$, the amount of analytes displaced was up to $35 \%$ compared to the $80 \%$ observed for D-DI and HS extractions. It is worth noting, however, that in real life applications of in vivo SPME, short extractions times, rather than equilibrium extraction, may provide well-balanced and unbiased coverage, of course, given that appropriate instrumental sensitivity is ensured. In addition, being that SPME is a technique sensitive to changes in matrix composition and alteration of binding equilibria, it can be used to explore and detect the matrix saturation effect, especially in response to alterations to physiological conditions. In this study, matrix saturation was investigated by monitoring the amount of probe analytes extracted vs. the amount of saturating compound spiked $(\alpha-$ pinene). Obtained results and further considerations are shown in Supplementary Information (Section 1.8 and figures S4-S5).

\section{Conclusions}

The results obtained in our work demonstrate the usefulness of SPME as a suitable tool for characterization of the behavior of organic compounds in complex food matrices. Here, we provide a thorough discussion on extraction kinetics, as well as analyte-matrix and analyte-sorbent interactions, which are critical components to such investigations. Our results corroborate that the DI-SPME approach does not only minimize the occurrence of artefacts associated with coating saturation, but also provides more balanced coverage between more and less polar analytes. The later outcome facilitates the investigation of multicomponent biosystems, especially when maintenance of physiological integrity of 
the sample is critical, as in the case of in vivo sampling. In summary, during DI-SPME, the analyte distribution on the coating simply reflects the actual molecular distribution of unbound analytes present in the biosystem. This study also demonstrates how PDMS-modified matrix compatible coatings, recently developed in SPME technology, represent the best choice for direct extraction from complex matrices. Their enhanced capacity drastically prevents artefacts related to coating saturation compared to nonmodified coatings. Through a systematic investigation of coating saturation and consequent inter-analyte competitive adsorption, critical parameters that needed to be carefully set during the application of SPME to complex matrix studies were established. In addition, by using high concentrations of probe molecules, the displacement phenomenon was used to gain insight into the selectivity of the coating and the analyte interactions taking place in solid porous coatings. In particular, DVB coating, in addition to displaying competitive adsorption based on the polarity of the analytes and their affinity for the coating, also showed the possible involvement of molecular recognition at the adsorption active sites. The analogue approach can be used to study the selective adsorption properties of macromolecules in the presence of competing compounds, using SPME at short sampling conditions when the displacement effects are not observed (see Figure 4). These findings open a new potential towards characterization of adsorbents and solid porous surfaces by using SPME in conjunction with the analysis of adsorption properties of macromolecules or suspended solids in biological matrices (eg. plasma, blood and other biological fluids) that regulate free/bound concentration of endogenous and/or exogenous compounds.

\section{AUTHOR INFORMATION}

\section{Corresponding Author}

*janusz@sciborg.uwaterloo.ca

\section{Funding Sources}

Natural Sciences and Engineering Research Council of Canada (NSERC) and Sigma-Aldrich Corporation.

\section{ACKNOWLEDGMENT}

E.G. thanks European Social Fund and Calabria Region for providing her fellowship, Dr. Erasmus Cudjoe and Ms. Nathaly Reyes-Garcés for the helpful scientific discussions and support and Prof. Giovanni Sindona and Prof. Antonio Tagarelli from University of Calabria.

\section{ABBREVIATIONS}

SPME, Solid-Phase Microextraction; DI, direct immersion: HS, headspace; DVB, divinylbenzene; Car, carboxen; PDMS, polydimethylsiloxane

\section{ASSOCIATED CONTENT}

\section{Supporting Information Available.}

Experimental details and additional figures as noted in the text. This material is available free of charge via the Internet at http://pubs.acs.org. 


\section{REFERENCES}

(1) Arthur, C. L.; Pawliszyn, J. Anal. Chem. 1990, 62, 2145-2148.

(2) Bojko, B.; Cudjoe, E.; Gómez-Ríos, G. A.; Gorynski, K.; Jiang, R.; Reyes-Garcés, N.; Risticevic, S.; Silva, É. A. S.; Togunde, O.; Vuckovic, D.; Pawliszyn, J. Anal. Chim. Acta 2012, 750, 132151.

(3) Cudjoe, E.; Togunde, P.; Pawliszyn, J. Bioanalysis 2012, 4, 2605-2619.

(4) Souza-Silva, É. A.; Lopez-Avila, V.; Pawliszyn, J. J. Chromatogr. A 2013, 1313, 139-146.

(5) Gionfriddo, E.; Naccarato, A.; Sindona, G.; Tagarelli, A. Anal. Chim. Acta 2014, 835, 37-45.

(6) Gionfriddo, E.; Naccarato, A.; Sindona, G.; Tagarelli, A. Anal. Chim. Acta 2012, 747, 58-66.

(7) Bojko, B.; Wąsowicz, M.; Pawliszyn, J. J. Pharm. Anal. 2014, 4, 6-13.

(8) Bojko, B.; Cudjoe, E.; Pawliszyn, J.; Wasowicz, M. TrAC Trends Anal. Chem. 2011, 30, 15051512.

(9) Bojko, B.; Reyes-Garcés, N.; Bessonneau, V.; Goryński, K.; Mousavi, F.; Souza Silva, E. A.; Pawliszyn, J. TrAC Trends Anal. Chem. 2014, 61, 168-180.

(10) Cudjoe, E.; Bojko, B.; de Lannoy, I.; Saldivia, V.; Pawliszyn, J. Angew. Chem. Int. Ed. Engl. 2013, 52, 12124-12126.

(11) Pawliszyn, J. In Handbook of Solid Phase Microextraction; 2012; pp. 61-97.

(12) Risticevic, S. Univ. Waterloo, PhD Thesis 2012.

(13) Souza Silva, E. A.; Risticevic, S.; Pawliszyn, J. TrAC Trends Anal. Chem. 2013, 43, 24-36.

(14) Vuckovic, D.; Risticevic, S.; Pawliszyn, J. Angew. Chem. Int. Ed. Engl. 2011, 50, 5618-5628.

(15) Contini, M.; Esti, M. Food Chem. 2006, 94, 143-150.

(16) Roberts, D. D.; Pollien, P.; Milo, C. J. Agric. Food Chem. 2000, 48, 2430-2437.

(17) Belliardo, F.; Bicchi, C.; Cordero, C.; Liberto, E.; Rubiolo, P.; Sgorbini, B. J. Chromatogr. Sci. 2006, 44, 416-429.

(18) Dunemann, F.; Ulrich, D.; Boudichevskaia, a.; Grafe, C.; Weber, W. E. Mol. Breed. 2009, 23, 501521.

(19) Zhang, Y.; Zhang, J. Anal. Chim. Acta 2008, 627, 212-218.

(20) Bianco, G.; Novario, G.; Zianni, R.; Cataldi, T. R. I. Anal. Bioanal. Chem. 2009, 393, $2019-2027$. 
(21) Setkova, L.; Risticevic, S.; Pawliszyn, J. J. Chromatogr. A 2007, 1147, 213-223.

(22) Souza-Silva, E. A.; Pawliszyn, J. Anal. Chem. 2012, 84, 6933-6938.

(23) Feinberg, M. J. Chromatogr. A 2007, 1158, 174-183.

(24) Risticevic, S.; DeEll, J. R.; Pawliszyn, J. J. Chromatogr. A 2012, 1251, 208-218.

(25) Vuckovic, D.; Cudjoe, E.; Musteata, F. M.; Pawliszyn, J. Nat. Protoc. 2010, 5, 140-161.

(26) Pawliszyn, J.; Musteata, F. M. In Handbook of Solid Phase Microextraction; 2012; pp. 383-397.

(27) Musteata, F. M.; Pawliszyn, J.; Qian, M. G.; Wu, J.; Miwa, G. T. J. Pharm. Sci. 2006, 95, 17121722.

(28) Vuckovic, D.; Pawliszyn, J. J. Pharm. Biomed. Anal. 2009, 50, 550-555.

(29) Pörschmann, J.; Kopinke, F.-D.; Pawliszyn, J. J. Chromatogr. A 1998, 816, 159-167.

(30) Bojko, B.; Vuckovic, D.; Pawliszyn, J. J. Pharm. Biomed. Anal. 2012, 66, 91-99.

(31) Górecki, T.; Yu, X.; Pawliszyn, J. Analyst 1999, 124, 643-649.

(32) http://www.chemspider.com/Chemical-Structure.74205.html.

(33) Guichard, E. Biotechnol. Adv. 2006, 24, 226-229.

(34) Górecki, T.; Yu, X.; Pawliszyn, J. Analyst 1999, 124, 643-649.

(35) Pawliszyn, J.; Shirey, R. E. In Handbook of Solid Phase Microextraction; 2012; pp. 99-133.

(36) Gimbert, F.; Morin-Crini, N.; Renault, F.; Badot, P.-M.; Crini, G. J. Hazard. Mater. 2008, 157, 3446.

(37) Mironov, N. a; Breus, V. V; Gorbatchuk, V. V; Solomonov, B. N.; Haertlé, T. J. Agric. Food Chem. 2003, 51, 2665-2673.

(38) Vuckovic, D.; Cudjoe, E.; Musteata, F. M.; Pawliszyn, J. Nat. Protoc. 2010, 5, 140-161.

(39) Bojko, B.; Gorynski, K.; Gomez-Rios, G. A.; Knaak, J. M.; Machuca, T.; Cudjoe, E.; Spetzler, V. N.; Hsin, M.; Cypel, M.; Selzner, M.; Liu, M.; Keshjavee, S.; Pawliszyn, J. Lab. Invest. 2014, 94, 586-594.

(40) Bojko, B.; Pawliszyn, J. Bioanalysis 2014, 6, 1227-1239.

(41) Lopes Da Silva, J. A.; Castro, S. M.; Delgadillo, I. J. Agric. Food Chem. 2002, 50, 1976-1984.

(42) Savary, G.; Guichard, E.; Doublier, J. L.; Cayot, N. Food Res. Int. 2006, 39, 372-379. 
(43) Savary, G.; Guichard, E.; Doublier, J. L.; Cayot, N.; Moreau, C. J. Agric. Food Chem. 2006, 54, 665-671.

(44) Zafeiropoulou, T.; Evageliou, V.; Gardeli, C.; Yanniotis, S.; Komaitis, M. Food Hydrocoll. 2012, 28, 105-109.

(45) Arvisenet, G.; Voilley, A.; Cayot, N. J. Agric. Food Chem. 2002, 50, 7345-7349.

(46) Arvisenet, G.; Le Bail, P.; Voilley, A.; Cayot, N. J. Agric. Food Chem. 2002, 50, 7088-7093. 
FOR TOC ONLY

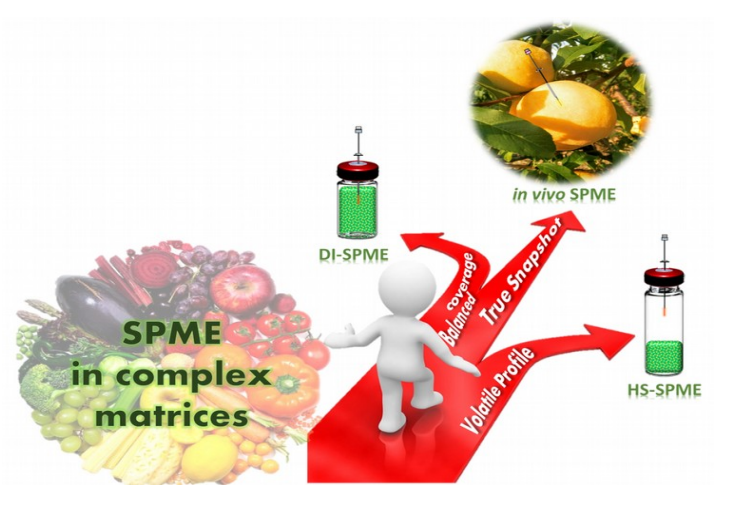

